\title{
Mémoires de l'esclavage à la Martinique
}

L'explosion mémorielle et la révélation de mémoires anonymes

Memories of Slavery in Martinique. Eruption of Memories and the Revelation of Anonymous Ones

\section{Christine Chivallon}

\section{OpenEdition \\ Journals}

Édition électronique

URL : http://journals.openedition.org/etudesafricaines/15847

DOI : 10.4000/etudesafricaines. 15847

ISSN : 1777-5353

Éditeur

Éditions de l'EHESS

\section{Édition imprimée}

Date de publication : 30 mars 2010

Pagination : 235-261

ISBN : 978-2-7132-2251-1

ISSN : 0008-0055

Référence électronique

Christine Chivallon, «Mémoires de l'esclavage à la Martinique », Cahiers d'études africaines [En ligne], 197 | 2010, mis en ligne le 10 mai 2012, consulté le 30 avril 2019. URL : http://

journals.openedition.org/etudesafricaines/15847 ; DOI : 10.4000/etudesafricaines.15847

Ce document a été généré automatiquement le 30 avril 2019

(c) Cahiers d'Études africaines 


\title{
Mémoires de l'esclavage à la Martinique
}

\author{
L'explosion mémorielle et la révélation de mémoires anonymes \\ Memories of Slavery in Martinique. Eruption of Memories and the Revelation of \\ Anonymous Ones
}

Christine Chivallon

1 La frénésie mémorielle autour de l'esclavage et de la colonisation, amplifiée au cours de la dernière décennie, a confronté la recherche sur l'espace français des «vieilles colonies » esclavagistes, à une difficulté majeure, celle de la connaissance des mémoires collectives fabriquées dans les mailles d'un dispositif narratif officiel bien mieux connu, celui de l'État français. Le consensus se fait désormais assez large pour affirmer que la mémoire produite par l'appareil colonial et continuée dans les formes institutionnelles de la dépendance a d'abord opéré par une longue oblitération, puis, bien plus récemment, par une acceptation avec ce point d'orgue que constitue la « loi Taubira » de 2001 «tendant à la reconnaissance par la République française de la traite négrière transatlantique et de l'esclavage en qualité de crime contre l'humanité " (Comité pour la mémoire de l'esclavage $2005: 15)$. Ce contexte de résurgence se prête à des interprétations confuses tant il laisse croire à l'arrivée subite d'une mémoire «neuve » dont la réception accrue, en tant que mémoire réinventée récupérant les référents lourds du passé pour servir l'action d'aujourd'hui, s'est opérée au moyen de l'usage de la catégorie d'identification «descendants d'esclaves» absente jusque-là des positionnements identitaires habituels. Envahi par des modalités nouvelles de dire l'héritage esclavagiste dans l'espace public, le champ mémoriel n'en apparaît que plus livré à l'incertitude d'en déceler les diverses composantes, et de distinguer, non pas bien sûr une "mémoire authentique " de l'esclavage, mais des mémoires formées dans les interstices de ces publicisations successives et excessives. Des mémoires qui auraient précédé l'affolement commémoratif souvent envisagé sous l'angle exclusif de son instrumentalisation politique ${ }^{1}$ et dont le récit qu'elles composent viendrait effectivement témoigner de l'esclavage comme référent explicite. Qu'en est-il en définitive des représentations du passé pour ceux qui ne sont pas les acteurs experts de la construction mémorielle et historique, mais les 
membres de groupes localisés au sein des sociétés antillaises, évoluant dans l'intimité des cadres de l'expérience reliée au passé esclavagiste? Hors du tapage mémoriel ayant dérivé en "guerres des mémoires » et prompt à faire naître le soupçon d'une mémoire mineure ${ }^{2}$, est-il possible d'identifier la trame de récits collectifs partagés, construits en contrepoint d'une injonction d'oubli massivement imposée et aujourd'hui appelée à laisser place à la reconnaissance dont la légitimité ne semble pourtant pas acquise ${ }^{3}$ ?

Cet article se propose d'affronter, pour les Antilles françaises, la question de l'existence possible de mémoires de l'esclavage bien moins redevables de processus circonstanciés liés à l'actualité des "retours du colonial» que de trajectoires intergénérationnelles porteuses de souvenirs sans cesse retravaillés. L'explosion mémorielle offre le paradoxe de l'apparition de catégories et de discours neufs tout en fournissant l'occasion d'une parole comme délivrée, facilitée dans sa propension à dévoiler des configurations anciennes restées discrètes et calfeutrées. Le propos qui suit prend appui sur ces surgissements qu'une recherche conduite au cours des années 2003 à 2007 à la Martinique a permis de saisir, comme si un tabou levé autorisait désormais à approcher ces "petits récits" produits dans l'intimité des familles et des collectifs restreints. Il s'agit plus précisément de rendre compte de discours construits à partir d'un événement ancien l'Insurrection du sud de 1870 - par les descendants directs des protagonistes, de ce qui fut une tentative de renversement de l'ordre colonial toujours structuré, vingt-deux années après l'abolition, par le clivage socio-racial propre à l'édifice esclavagiste. Le recueil de ces récits nous invite à travailler dans deux directions. La première est celle du sens porté par ces discours en rapport avec la portée de la violence annihilante induite par le traitement répressif auquel a donné lieu l'insurrection. Il indique l'existence d'interprétations bâties en contrebande pour sauver l'intégrité de soi et extraire le corps familial de l'anéantissement que fait encourir l'héritage d'une identité rendue coupable de crimes par la justice coloniale. La seconde direction est celle de la présence de ces discours au sein de la société martiniquaise actuelle quand ceux-là ont été privés des outils d'une symbolisation portée par les lieux de l'autorité sociale légitime. Ces dispositifs narratifs où la focale se resserre sur l'esclavage au travers d'événements très localisés renouvellent la question des mémoires collectives à la Martinique en dévoilant des « témoins » et interrogent la nature de cette trace mémorielle dans son rapport à la véracité historique et à la fidélité au souvenir, comme dans sa possibilité à formuler tout autant qu'à transmettre des contre-représentations.

\section{Mémoires absentes ou mémoires invisibles?}

2 Dans l'abondant corpus théorique disponible sur la mémoire, et de manière à bien situer de quelles mémoires il s'agit pour notre propos, on se limitera seulement à convoquer l'apport de Paul Ricœur (2000) et celui de Maurice Halbwachs (1997). Au premier, nous emprunterons une approche de la mémoire qui se trouve incorporée à la constitution de l'identité au travers de la fonction narrative par le rapport au temps que cette fonction instaure en conjonction avec l'évaluation du présent et la projection du futur. La mise en récit de l'identité est toujours tendue vers le maintien de soi dans le temps et se trouve ainsi instituante d'une éventuelle "mêmeté " temporelle dont l'exclusive identitaire a prouvé savoir se saisir (Ricœur 2000 : 98-103). Elle s'offre donc comme possibilité de la continuité de soi et du même en figurant, par la représentation, la présence d'une chose paradoxalement toujours absente : le passé. C'est ce passé configuré qui sert l'actualité du 
lien social, par l'entour identitaire qu'il conforte pour ceux qui en partagent le sens et se trouvent associés dans ce «vivre-ensemble» nécessairement localisé dans une temporalité commune. Hors de cette généralité sur l'identité narrative qui construit la mémoire, nous retiendrons particulièrement pour notre approche le double statut qu'assigne Ricœur au souvenir, en tant que chose apparaissant presque passivement, et en tant que chose objet d'une quête, intégrée ou rappelée volontairement à la mémoire. Cette intervention pragmatique sur les « images » du passé incite le philosophe à postuler le dédoublement de deux intentionnalités. L'une, produit de l'imaginaire ayant la capacité de faire être à l'esprit ce qui n'existe pas, est dirigée vers le fantastique. L'autre, produit de la mémoire cognitive, ayant la capacité de rappeler ce qui a existé, est tendue vers la réalité antérieure (ibid. : 53-54). Le découplage quasi impossible de ces deux opérations, n'en reste pas moins l'argument fort qui fait affirmer au philosophe qu'en dépit de la menace permanente de confusion entre remémoration et imagination, «nous n'avons pas mieux que la mémoire pour assurer que quelque chose s'est passé avant que nous en formions le souvenir » (ibid. : 7). C'est pourquoi, la mémoire est forcément conçue comme la matrice de la représentation historienne (ibid. : 106, 354-367).

Nous nous référerons au second auteur qui nous sert de guide, Maurice Halbwachs, pour la distinction qu'il a opérée - toujours vue comme irremplaçable dans une large sphère de spécialistes (Kansteiner: 2002) - entre "mémoire collective» et "mémoire historique » (Halbwachs 1997). Rappelons brièvement que la première est définie comme « un courant de pensée continu, d'une continuité qui n'a rien d'artificiel puisqu'elle ne retient du passé que ce qui en est encore vivant ou capable de vivre dans la conscience du groupe qui l'entretient» (ibid.: 131). La seconde procède de la rupture. Elle indique au sein d'un même ensemble, la succession générationnelle de groupes séparés tels « deux tronçons en contact par leurs extrémités opposées » (ibid. : 132) : elle suppose le recours à l'artifice, notamment celui de l'archive, pour reconstruire a posteriori un passé dont les représentations ne circulent plus. La mémoire historique est de l'ordre d'une posture de l'écart, de la distanciation, du retrait, qui récupère le passé pour le nommer, le qualifier et même le commémorer, alors que la mémoire collective est de l'ordre d'un en-dedans qui ne cherche pas le passé, mais en transmet la trame significative. Et de la même manière que Paul Ricœur tout en postulant la perméabilité extrême de la mémoire et de l'imagination voulait cependant les séparer, on peut dire que Maurice Halbwachs tout en opposant radicalement histoire et mémoire, laisse entrevoir, ne serait-ce que par le choix du terme "mémoire historique», que la mémoire - collective ou historique - se constitue selon des registres qui s'entremêlent sans forcément être étanches, ni dans le temps, ni dans l'espace.

4 C'est semble-t-il cette "mémoire historique » qui s'est généralisée dans le champ mémoriel, portée désormais par un large ensemble d'acteurs non limité aux cercles intellectuels traditionnellement producteurs du discours historicisant sur le passé esclavagiste. Les travaux de Marie-José Jolivet (1987) ont très bien montré combien cette mémoire historique à la Martinique, dans sa quête de sens tendue vers la dénonciation des errements esclavagistes, avait fini par disqualifier la mémoire collective, la faisant être par sa pauvreté postulée, l'une des conséquences majeures de l'horreur de l'esclavage. Les écrits d'Édouard Glissant maintes fois repris ont formé l'armature de cette représentation "réflexive » où la mémoire collective apparaissait comme terriblement incomplète, « raturée ", incapable de sédimenter le corps social, comme stabilisée " dans le néant d'une non-histoire imposée » (Glissant 1981: 67-69; 131-133). Ils se font l'écho 
d'une dissociation nette entre la mémoire historique et la mémoire collective, celle-là se trouvant investie de la mission de développer une "vision prophétique du passé " pour un peuple privé de sa «densité collective» (ibid.: 132). La mémoire historique plus récente, développée par les milieux associatifs et militants opèrent par « réconciliation " de ces deux mémoires (historique et collective), chargeant la catégorie "descendants d'esclaves » largement mobilisée - y compris désormais par Édouard Glissant (2007 : 138) pourtant peu sensible aux modèles de l'identité - de reconstruire une continuité intergénérationnelle ${ }^{4}$. Dans les deux cas pourtant, cette mémoire historique - qu'elle considère le néant mémoriel du collectif ou qu'elle fabrique et théorise une continuité nouvelle - procède bien de la rupture qui reconfigure la teneur d'un passé réinvesti, laissant entière la question de l'existence de mémoires collectives redevables d'un " courant de pensée continue ». C'est ce constat que la vindicte anti-mémorielle ${ }^{5}$ est prête à tenir pour preuve d'un rapport essentiellement instrumental au passé, puisqu'il réinvente la filiation perdue avec les victimes de l'esclavage. Il informe pourtant sur la diversité des registres mémoriels dont la légitimité de l'usage ne devrait pas se mesurer à l'aune de la fidélité à une quelconque tradition ou de la place généalogique dûment attestée, mais à celle de la pertinence de la problématique portée dans le champ politique : ici un rapport non résolu à une histoire éminemment conflictuelle dont les échos se font entendre dans l'actualité des rapports sociaux ${ }^{6}$.

Dans le bilan récent dressé sur les études relatives à la mémoire de l'esclavage (Chivallon : à paraitre) qu'il n'est pas le lieu de détailler ici, il est apparu que la recherche sur les Antilles françaises s'est trouvée prise dans une sorte d'étau. D'un côté, elle a rencontré la tendance propre à l'anthropologie des Amériques noires structurée par une « épistémologie de la vérification » comme a si bien su la nommer David Scott (1997: 21) et destinée à valider ou non la présence d'un héritage africain qui aurait survécu par-delà le transbordement et l'asservissement. De ce point de vue, la typologie des communautés noires des Amériques qu'offrait Roger Bastide (1967) se fait exemplaire de cette tension vers l'identification des formes culturelles redevables à l'expérience ante-esclavagiste portée depuis l'Afrique ancestrale. De l'autre, elle s'est heurtée au paradigme dominant pour la Caraïbe française que l'on vient d'entrevoir et qui ne consent à reconnaître que dépossession et incomplétude dans les formations sociales des anciens esclaves. Dans cette perspective, l'esclavage a été éradication et impossibilité de reconstruction. Comment peut-on dès lors concevoir que des mémoires aient pu "témoigner", par le souvenir reconduit, d'une telle expérience et en construire le sens, alors que les rouages mêmes de la construction mémorielle ont été conçus comme inopérants? Si la recherche n'a pas déserté, loin de là, la thématique mémorielle, elle l'a pourtant envisagée sous cet angle particulier qui lui a fait rendre compte de l'héritage de l'esclavage dans la manière dont il s'est trouvé signifié par des formes implicites ou inconscientes et si ce n'est, à l'extrême, par des formes déstructurées ou aliénées. D'où la conclusion de ce bilan (Chivallon à paraitre) : soit l'esclavage est resté un non-dit recouvert par une symbolique de la référenciation diffuse et dans ce cas aucun récit - ni petit de l'ordre de l'individuel, ni grand de l'ordre du meta-récit collectif - n'existe et seuls persistent des détournements de sens ; soit l'esclavage a pu se dire, se dit encore, mais le chercheur en a sous-estimé la possibilité pour des raisons bien plus imputables à l'histoire des idées qu'à une quelconque absence de discernement.

Le contexte actuel avec la polarisation qu'il occasionne sur la mémoire - plutôt historique - construit justement un nouveau milieu d'exploration en raison non seulement d'une migration des paradigmes, mais aussi d'une levée de certains tabous 
concernant autant les chercheurs que les «témoins ». Ce contexte met de plus en plus en avant la nécessité d'envisager une multiplicité de registres mémoriels produits simultanément au sein d'un même collectif, à commencer par les registres de la " gouvernementalité ». Ces derniers nous apparaissent plus clairement avec un ensemble de travaux d'historiens qui convergent pour attester « d'une politique de l'oubli » mise en place dès les lendemains de l'abolition de l'esclavage, relayée par la bourgeoisie de couleur qui en a fait son "leitmotiv" pour promouvoir son aspiration d'assimilation égalitaire dans les cadres coloniaux (Reinhardt 2006: 123). Décrite comme «le levier puissant » du contrôle post-esclavagiste (Schmidt 1999: 454), cette politique visait à accréditer l'idée d'une société neuve ayant fait œuvre d'expiation des miasmes de l'esclavage alors que les rapports sociaux étaient restés articulés selon les mêmes clivages socio-raciaux (Cottias : 2006). L'avancée républicaine a continué de se fondre dans ce cadre idéologique donnant au décret d'abolition une dimension hautement symbolique, celle d'un acte fondateur de la République, encore réclamé comme tel par le Président Jacques Chirac lors du cent-cinquantenaire de l'abolition en 1998. Si l'esclavage est parvenu à intégrer le récit national, ce n'est donc que sous la forme paradoxale de son existence anéantie ${ }^{7}$.

La question de cet oubli prôné officiellement et qui n'est qu'une manière de rendre silencieux des pans entiers de l'expérience antillaise ajoute à la difficulté d'identifier des dispositifs narratifs dès lors enfouis, à la manière des processus décrits par Michel-Rolph Trouillot (1995) en rapport avec la révolution haïtienne. Car si l'histoire se révèle toujours au travers de la production de récits, ceux-là sont le résultat « de l'exercice différentiel du pouvoir qui rend possibles certaines narrations et réduit au silence les autres » (ibid. : 25). Pour la Martinique, "l'histoire» des mémoires collectives serait peut-être à considérer comme celle d'une confusion entre absence et invisibilité. «L'oubli » a été le trope d'une narration imposée dont il reste à aborder les conséquences qu'elle a eues, soit qu'elle ait définitivement grippé les ressorts de la conscience collective comme le laissaient entendre les écrits sur la "dépossession »; soit qu'elle ait pu converger avec une volonté populaire d'oublier le passé, par « refus citoyen de l'esclavage » comme le propose Michel Giraud (2005: 544); soit encore qu'elle ait rendu inaudibles des voix porteuses d'autres visions du passé. Cette troisième voie est celle que l'anthropologue Richard Price, pourtant familier de récits de mémoire d'une forte densité comme ceux qu'il a collectés au Surinam auprès des Marrons Saramaka (Price 1994) a choisi de suivre. L'oblitération ancienne, la folklorisation galopante, l'hyper-modernisation, la puissance de l'assimilation «voilent à peine l'élément de résistance qui n'est jamais loin de la conscience populaire» (Price 2000 : 181). Les descendants des insurgés de 1870, dont la filiation n'est pas soudainement réinvestie, disent cette résistance au travers du «souvenir » d'une réalité antérieure douloureuse dont les générations successives ont transmis et accommodé les « images » à mi-chemin entre imaginaire et remémoration.

\section{Retour historiographique sur un événement majeur : I'Insurrection du sud de 1870}

6 Dire de l'Insurrection du sud de $1870^{8}$ qu'elle a été un événement majeur contredit la représentation qu'elle occupe dans l'historiographie martiniquaise où elle rejoint le lot des révoltes sporadiques ordinaires. C'est là la conséquence directe du traitement qu'elle a reçu, ce que Gilbert Pago, l'un des très rares historiens à s'être intéressé à cet 
événement, avait pressenti en parlant de l'oubli des historiens comme la conséquence logique d'une propagande officielle destinée à exorciser ce qui n'était ni plus ni moins qu'un "acte digne d'un pays de sauvages» (Pago 1974: 1). La formule "pays de sauvages » n'a rien d'abusive puisque tout au long du procès qui jugera les insurgés, le couple «civilisé/sauvage» sera utilisé sans modération. Cette insurrection, appelée localement "affaire Codé ", éclate immédiatement après la proclamation de la Troisième République, en septembre 1870, à Rivière-Pilote, commune du Sud de l'île. C'est là que réside le planteur blanc Codé, ouvertement conservateur, et assesseur quelque temps auparavant au procès d'un jeune Noir condamné à cinq ans de bagne pour avoir frappé un Blanc qui l'avait humilié. L'iniquité de cette peine - surtout quand elle est comparée à celle encourue par les Blancs à la même époque pour des faits d'une plus grande gravité provoque une émotion vive au sein des populations noires. Mais ce jugement n'est que l'étincelle qui précipite les événements. Ceux-là trouvent leur source profonde dans le contexte de ces années post-abolitionnistes marquées par la mise en place d'un système extrêmement répressif à l'égard des anciens esclaves libérés en 1848, pour les contraindre à réintégrer le travail sur les plantations et faire obstacle au mouvement d'installation d'une paysannerie dans les régions des hauteurs de l'île. L'arrêté du gouverneur Gueydon sous le Second Empire forme le point culminant de cette police du travail rendu obligatoire sous la menace de la « contrainte par corps ». Les mots de Victor Schoelcher (1872: 10-17) ont résumé mieux que tout autre la situation créée par « un abominable arrêté ", caractérisé par « une justice distributive » faite "d'abus », de "vexations », de «tyrannies ", « d'inquisition » et "d'oppression » qui conduisent à la perte de « la libre disposition de soi-même", tout ceci au bénéfice des "grands défenseurs de l'ordre aux colonies [qui] ne veulent pas que la loi atteigne certains privilégiés ». Dans ce contexte de quasi retour à l'esclavage, l'édifice social dans son entier reste dépendant de la structuration par l'appartenance raciale. C'est pourquoi, quand la République est proclamée, la population de Rivière-Pilote l'acclame aux cris de « Vive la République » et de « Mort à Codé ». L'assemblage de cette ovation républicaine avec l'exhortation à tuer les Blancs condense ce qui pour l'instant n'est qu'un horizon d'attente exprimé de manière cathartique : la disparition de la caste béké 9 pour une République pressentie comme incompatible avec «l'injustice des Blancs» selon les mots prêtés à l'insurgé Auguste Villard au cours du procès ${ }^{10}$.

7 L'insurrection dure à peine une semaine. De nombreuses bandes de 300 à 600 personnes se forment dans toutes les communes du sud. Un camp fortifié est organisé dans les hauteurs de Rivière-Pilote, sur les terres d'Eugène Lacaille, petit propriétaire mulâtre. Avec lui, Louis Telga, autre chef insurrectionnel, petit propriétaire noir, tente de transformer en une armée les cultivateurs des plantations environnantes et les paysans, munis de piques, de coutelas, de bouteilles emplies d'eau pimentée, d'armes à feu dérobées. Pour autant, même si elle laisse entrevoir des figures majeures, l'insurrection est conduite au sein de groupes formés spontanément comme si cette liberté aspirait à se passer de chefs patentés, ce que dit à sa manière l'insurgé Cyrille Micamor, lors du procès : « Personne n'a forcé personne ; c'était la première fois que ça arrivait ; c'était la République ; on disait que tout était permis. »

8 Incendies à la torche la nuit, désertion des habitations, pillages, terreur, menaces, affrontements sanglants avec l'armée coloniale, traque de Codé... sont le lot des jours qui suivent la proclamation de la République. L'état de siège est déclaré par le gouverneur. Les troupes régulières ou volontaires viennent à bout des bandes, encerclent, tuent ou 
capturent les insurgés. Le bilan de la répression ne sera jamais connu mais le nombre des prisonniers est plus certain : 500 hommes et femmes sont enfermés au fort militaire à Fort-de-France. Le gouverneur annonce «la victoire», loue le "patriotisme» et le concours de «toutes les populations ». Dans le rapport qu'il rédige sur les événements, il affirme que ce mouvement insurrectionnel a été « le plus terrible que nos colonies aient connu depuis la révolte de Saint-Domingue » (Menche de Loisne 1931:20). Les actes du procès laissent effectivement transparaître l'esquisse d'un projet où l'on rapporte qu'Eugène Lacaille voulait installer un nouvel ordre, sans Blancs, " pour laisser le pays aux nègres et aux mulâtres » et "effectuer le partage des terres ». Mais le mouvement déborde largement toute planification. Il est avant tout explosion d'une frustration trop longtemps contenue pour laquelle la proclamation de la République ouvre la brèche en s'offrant cependant dans une contradiction intenable: celle d'être portée par l'autorité coloniale déjà en place. Car l'annonce officielle de cette République faite par le gouverneur se fait, non pas sur le mode du passage bienvenu à un ordre politique nouveau, mais sur celui de l'intimation à ce que rien ne change.

L'appel à tuer "Codé " n'en reste pas au stade de la liquidation verbale des affects refoulés. Le passage à l'acte se produit quand un groupe d'insurgés finit par retrouver celui que l'on appelle encore le « maître » au détour d'une phraséologie mal maitrisée par les membres du tribunal. L'assassinat s'accomplit collectivement par une bande où ni Telga, ni Lacaille ne sont présents. L'homme est massacré, frappé de cinquante coups de coutelas, ses plaies arrosées de liquide pimenté. La tradition orale veut qu'il ait été émasculé : ultime geste d'une vengeance portée à son comble où le corps se charge de dire par la violence des mutilations que le pouvoir du maître, celui ancestral dont on dit qu'il repose sur le viol originel des femmes noires captives ${ }^{11}$, ne pourra plus s'établir. Dans le sang de Codé, un mouchoir est trempé, arboré tel un étendard par un de ses anciens "serviteurs » (que Codé avait par ailleurs fait comparaître au tribunal, pour des raisons inconnues) comme si le sang ne pouvait être que la réponse à l'arrogance menaçante du drapeau blanc esclavagiste que le planteur continuait de faire flotter sur son "Habitation $»^{12}$. Cette violence dit à elle-seule l'ampleur de l'atteinte de soi qui l'a suscitée, dans cette douleur infligée qui accomplit l'espace d'un moment un retournement du «fantasme de la toute-puissance » du groupe qui détenait jusque-là le «bénéfice politique » des instruments d'oppression et d'humiliation, tant il est vrai, selon les mots de David Le Breton (2006: 197) que «la puissance d'un homme ou d'un État se mesure à la somme des douleurs qu'il est susceptible de prodiguer sans qu'aucune de ses prérogatives ne soient encore menacées par la rebuffade des victimes ou la rigueur de la loi ».

Le procès qui suit l'insurrection au cours de l'année 1871 s'applique à faire de l'assassinat de Codé le point névralgique de ce qui finit par être la transfiguration d'une tentative de révolution en crime odieux. Dans un univers culturel particulièrement sensible aux rites qui entourent la mort et aux soins qui accompagnent le départ d'un défunt, l'acharnement sur le corps de Codé devenait en soi suffisamment efficace pour faire passer au second plan toute autre dimension de cet acte insurrectionnel, efficacité qui n'a pas échappé aux membres du tribunal qui n'ont eu de cesse de polariser l'attention sur la mort du planteur. Les réquisitoires et plaidoiries, à aucun moment, ne suggèrent les conditions de vie des insurgés comme ayant pu entraîner une révolte. Seul le crime sur la personne demeure. Cette transfiguration, en puisant sans modération dans le registre religieux, s'accompagne de la béatification de la figure de Codé. Le parcours de souffrance 
du colon se voit intégré à la trame de « la passion du Christ » dans un discours porté par l'ensemble des membres du tribunal qui du même coup tracent les contours des héros de ce moment colonial: tous ceux, serviteurs humbles et fidèles, qui ont aidé Codé à échapper à la barbarie des «hordes de sauvages ", héros qui seront par ailleurs décorés lors d'une cérémonie à Rivière-Pilote, en décembre 1871, une semaine à peine après l'exécution des cinq premiers condamnés à mort. La fusion entre Codé et la nation républicaine n'a plus qu'à s'opérer pour ravaler les insurgés au rang de traites à l'idéal républicain à partir d'une série d'équivalences opposant deux mondes : d'un côté, celui de la civilisation, de la propriété bienfaitrice, de la nation généreuse et glorieuse ; de l'autre celui de la sauvagerie, du refus du travail, de l'infamie et du parricide éhonté.

La seconde transfiguration que réussit le procès se fait au cours de l'aménagement d'un espace discursif particulier où les « races » sont proclamées ne plus exister. Les insurgés sont littéralement accusés d'avoir réveillé ce qui avait été "oublié» depuis l'acte d'abolition. Ainsi ont-ils exploité « les souvenirs irritants d'un passé à jamais disparu » et réveillé " des rancunes depuis longtemps sans cause ». C'est à la France que revient le mérite d'avoir su "étouffer toute suprématie privilégiée » : se réclamer de la République, c'est forcément s'inscrire dans la continuité des régimes successifs qui ont anéanti les clivages raciaux. Ainsi déclarée non avenue, la catégorie raciale peut se déployer encore plus librement. La désignation des groupes en fonction de leur appartenance raciale, la légitimité de leur place en fonction de cette même appartenance, transpirent effectivement des propos tenus tout au long de la procédure judiciaire jusque dans ce qui finit par devenir possible à énoncer tant il a été martelé le contraire : l'existence d'une «classe qui a fondé la colonie» et derrière laquelle «il y a la France pour la sauvegarder $»$.

Au terme de ce procès, tout à la fois répressif et éducatif, le jugement prononcé achève de construire une vision sociétale où la France ressort à la fois triomphante et généreuse, parce que capable de pardon. L'amnistie va ainsi être appliquée de manière discrétionnaire à tous ceux considérés avoir été des « enfants » abusés que la mère patrie concède à reprendre sous son aile pour " qu'ils grandissent » et "progressent vers une élévation sociale $»^{13}$. Pas question comme avec l'amnistie générale prononcée pour les révoltes de 1848 - au cours desquelles un meurtre comparable à celui de Codé avait été commis - de procéder à " une remise à zéro de l'histoire de la colonie » (Cottias 2007 : 30). Au contraire, il s'agit en 1870 d'inculquer que plus rien d'autre n'est à attendre d'une patrie aujourd'hui républicaine qui a déjà tout donné d'un ordre juste. Ce sont donc ceux présumés coupables qui se retrouvent sur les bancs des accusés. Pour eux, les condamnations les plus lourdes seront sans appel allant de la peine capitale aux travaux forcés à perpétuité et à la déportation en enceinte fortifiée.

D'un point de vue historiographique, on peut dire de ce moment colonial qu'il fait correspondre l'entrée dans la stabilité républicaine avec la définition d'une République qu'il convient de qualifier de "raciale ». Celle-là met en place un pacte particulier où l'aveuglement est requis pour sceller un lien quasi sacré avec ceux doués de la croyance en cet imaginaire colonial d'une égalité virtuelle, mettant violemment au ban de la société ceux qui ne sauraient y croire. 


\section{La vitalité de mémoires vagabondes : les descendants des insurgés ou les porteurs/créateurs de mémoire}

11 Pour retrouver les « descendants » des insurgés, la méthode a consisté à travailler à partir des patronymes relevés au travers des actes du procès et dont il était possible de postuler - à la suite de travaux antérieurs sur la paysannerie martiniquaise (Chivallon 1998) qu'ils avaient été associés à la constitution du patrimoine foncier caractéristique du secteur de la petite propriété. La recherche conduite au cadastre - pour la commune de Rivière-Pilote - a confirmé la présence maintenue jusqu'à nos jours, de certains de ces noms anciens, attestant de ce profil propre à la société rurale où les « quartiers » paysans des régions montagneuses portent encore la marque de leur fondation autour des familles " pionnières » d'anciens esclaves (ibid.). Les recherches plus intensives ont concerné un échantillon de onze patronymes: un nombre restreint en raison de l'objectif fixé qui consistait non seulement à travailler sur « l'actualité » des mémoires mais aussi sur son croisement avec l'histoire. Il s'est agi en effet de retracer les trajectoires des différents protagonistes autant par l'oralité que par la source écrite, ce qui a permis d'embrasser une large temporalité qui amène par exemple aux années 1720 , concernant la parenté du « chef » Eugène Lacaille. À titre indicatif, car la « comptabilité » n'était pas précisément l'objet de ce travail, on dira que pour huit de ces patronymes, les différentes sources utilisées ont conduit à rétablir le lien direct avec l'insurgé mentionné dans les actes, et les personnes rencontrées aujourd'hui, ce qui rend compte, non pas de l'existence révolue de tout lien pour les trois autres, mais des limites de cette recherche où il n'a pas toujours été possible de trouver les "témoins " proches dans le temps et dans l'espace. Parmi les dix-neuf personnes "ressources» rencontrées grâce au "bouche à oreilles $»^{14}$, onze avaient une connaissance très précise du lien avec l'aïeul insurgé (elles se rattachent à six des onze patronymes). On comprendra en fonction de l'économie de cet exposé que leurs témoignages ne pourront être présentés que de façon très fragmentaire, dépendante de surcroît de la surimposition de narrations, celles des témoins et du chercheur.

\section{Le silence et la honte en partage : mémoires douloureuses}

12 Ceux qui racontent connaissent donc leur filiation avec l'aïeul impliqué dans les événements de 1870, et plus encore, la teneur de l'héritage laissé au fil des presque 140 années qui les en séparent. Ils sont les arrières ou arrières arrière-petits-enfants des insurgés et forment ainsi la quatrième ou la cinquième génération depuis l'insurrection. Leurs récits convergent sur deux aspects qui charpentent la teneur de l'expérience véhiculée, le silence et la honte, le premier devenant un moyen de se défendre contre la seconde. L'insurrection n'est connue des mémoires que sous le nom « d'affaire Codé » où se lit immédiatement le filtre de lecture fabriqué à l'époque du procès : le meurtre du planteur. La honte relatée n'est pourtant pas celle de la conviction en l'accomplissement d'un acte irrémissible, mais celle du poids que fait peser sans relâche le regard de la société locale sur ceux considérés comme des fils d'assassins :

«À 88 ans, ma mère [arrière-petite-fille de l'insurgé] a encore ce sentiment de honte. Elle l'a parce que je pense que ça a terni un peu son enfance. Son enfance scolaire en tout cas. Elle s'est sentie pointée du doigt, méprisée, raillée parce que c'était comme ça : "la famille qui a tué 'Codé”. Et à la manière dont elle en parle, on sent qu'elle a beaucoup souffert, et qu'elle en souffre encore ».(Yolette J., 56 ans, 
institutrice, arrière arrière-petite-fille de Jean Vincent-Rosine, mort en prison en 1871, avant le procès, présent lors de l'assassinat de Codé)

«Ma maman m'a raconté une fois qu'il y avait un notaire au Marin [commune voisine de Rivière-Pilote] qui s'appelait Maître Saint-Cyr [...]. Lorsqu'il a su que ma maman allait se marier avec Constantin [Telga], il a dit: "ou ka mayé épi ich asasen" ["tu te maries avec un enfant d'assassin"] [...]. Je me rappelle, une fois, je travaillais à l'usine du Marin. Un gars m'a envoyé ça à la figure : "mé sé jénérasion zot ki koupé koko Kodé. Ki sa zot lé fé atchelmen" ["Mais, c'est votre génération qui a coupé le sexe de Codé. Qu'est-ce que vous voulez faire maintenant ?"]. Un camarade à moi qui m'a lancé ça à la figure ».(Constant Victor T., 83 ans, ancien ouvrier en bâtiment, arrière-petit-fils de Louis Telga, chef insurrectionnel, condamné à mort par contumace, enfui dans l'île de Sainte-Lucie. Aucun membre de la famille n'a su ce qu'il était devenu)

Les familles transportent ainsi l'expérience du déshonneur à l'intérieur de leur univers de sociabilité ordinaire. Un déshonneur qui peut ressurgir à n'importe quel moment, au travers de sentences brutales qui placent les individus dans un « à-côté » communautaire. Certains témoins disent qu'elles se prononçaient encore jusque dans les années 1960 "jusqu'à 14 ans, on m'appelait "fanmil brilé 'Kodé”" (" la famille qui a brûlé Codé ») avant que ne s'affirme l'entrée dans l'ère de la militance nationaliste marquée par l'arrivée d'Alfred Marie-Jeanne à la tête de la mairie de Rivière-Pilote, en $1971^{15}$. Dans leur enfance, certains ont participé à des processions organisées par le prêtre de la paroisse de Jossaud, le père Croquet de Béligny, d'origine béké. Le quartier « Jossaud » est tout proche du lieu où s'est déroulé le drame, à savoir au pied d'une croix, déjà présente en 1870 sur le terrain de Jean Vincent-Rosine, et nommée depuis "la Croix Codé », le toponyme continuant de lui-même à rendre présent à la conscience la vision sacrificielle de la mort $\mathrm{du}$ planteur. Les processions qui se déroulaient pendant le carême étaient vouées à l'expiation, apportant ainsi tout le crédit de l'autorité religieuse à la désignation d'une partie "impure " du corps social ayant souillé son âme par ses actes. La vision d'un événement vu unilatéralement comme relevant du démoniaque est à ce point intégrée qu'elle donne lieu à la peur d'une malédiction sans cesse menaçante : cent années après l'insurrection, en 1970, quand un incendie se propage dans le bourg de Rivière-Pilote, il est immédiatement interprété comme une punition divine. Dans ce contexte de peurs entretenues, la désignation des familles des insurgés en coupables impardonnables n'en est que plus forte. Elle nourrit le sentiment d'une atteinte profonde à la dignité et à l'honneur pour ceux qui restent ainsi stigmatisés. C'est autour du nom que se polarisent les affects contradictoires résultants de cette exclusion. Des descendants - ceux de la génération des enfants des insurgés - ont changé de noms pour tenter de survivre aux invectives : certains des descendants d'Eugène Lambert Lacaille ont réduit le patronyme aux seuls prénoms "Lambert » ou « Eugène ». Cette transformation patronymique s'est transmise comme une véritable amputation, une atteinte à l'intégrité du corps familial :

«Mon arrière-grand-père [Eugène Lacaille] a été poursuivi par la milice, par l'armée, par les gendarmes, la police locale. Il y a eu un procès, tout ça. Donc il y a eu beaucoup de mes parents qui se sont faits déportés ailleurs, c'est-à-dire qu'ils sont partis de la Martinique à cause du nom qui était sali [...]. Il y a des gens qui ont pris le prénom pour le nom [...]. Dans la mesure où c'était une espèce de tare de porter le nom Eugène [Lacaille] à l'époque. Surtout que l'on disait ces gens là ont la malédiction de Codé [...]. Donc on a condamné mon arrière-grand-père, comme ça. Et je pense que ça a joué beaucoup dans la famille. Ça c'est sûr. Toute la famille a été éparpillée. La famille Eugène [Lacaille] qui était une famille unie et prospère dans le temps, est devenue une famille séparée de partout ».(Liliane F., 62 ans, institutrice, 
arrière-petite-fille d'Eugène Lacaille, condamné à mort, petite-fille de Turiaff Lacaille condamné à vingt ans de travaux forcés)

«Donc, à ce moment là, l'acte est jugé comme odieux, comme inhumain. Et on se retourne contre lui [Jean Vincent-Rosine] au moment de trouver les coupables. Tous on a une réputation. Donc à partir de là, la famille Rosine, la grande famille Rosine dans laquelle Vincent-Rosine se trouve, l'extra-famille [...] estime que Vincent-Rosine a jeté l'opprobre et la honte sur la famille et on se débarrasse de son nom et c'est pourquoi la génération suivante, Jean Vincent, le fils, il n'est plus Rosine [...]. Nous avons dans notre famille des parents âgés et décédés [...] malgré l'histoire du nom perdu, ils savaient en fait qu'ils étaient des parents de sang. Donc le nom Rosine a disparu, mais il y a des descendants Vincent qui connaissent leur histoire, qui le savent. Et quelques éléments Rosine qui l'admettent, le reconnaissent et le disent [...]. Vincent en fait, c'est un nom. C'est pas le vrai nom ». (Yolette J., op. cit.)

L'item de la stigmatisation en «familles d'assassins » est commun à tous ceux à qui l'on a fini par énoncer des bribes de la trame de la tragédie. Car le silence s'est trouvé être un mode particulier de transmission: à la fois une manière de taire, au sein même des familles, l'existence connue des événements mais aussi une manière de dire, par touches, les repères majeurs construits comme un secret partagé à l'intérieur du cercle familial et rendu silencieux pour l'extérieur.

Le « taire » a été le propre de la génération immédiate ayant succédé à celle des insurgés, celle rescapée de la répression, comprenant des «amnistiés » et même des graciés de 1880, revenus du bagne, puis repartis en Guyane, comme Turiaff Lacaille, le grand-père de Liliane. De lui, Liliane ne connaît pas la condamnation au bagne mais seulement l'exil que lui a raconté son père, fils de Turiaff, et mis sur le compte de l'impossibilité à se reconstruire en Martinique :

«Le père de mon père [Turiaff] s'expatrie parce qu'il n'a plus d'avenir ici. Il ne peut gagner sa vie. Il est un proscrit. Il est persécuté [...]. Il faisait partie de l'insurrection aux côtés de son père. Il risquait d'être pris. Donc, il est parti en Guyane. Mon père ne m'a jamais dit dans quelles conditions [...]. Je pense que c'est par rapport à cette grosse douleur, ce gros problème qu'il y a eu dans la famille. Parce que c'est un ouragan qui balaye une famille. C'est pas un seul, c'est le père et tous les autres fils qui étaient avec leurs femmes ».(Liliane F., op. cit.)

Les plages de ce silence transmis que les témoins d'aujourd'hui racontent, intègrent cette "période" de latence nécessaire au collectif avant de permettre le retour du traumatisme enfoui (Fassin \& Rechtman 2007: 31-32; Eyerman 2008: 33). L'étude des mémoires constituées à la suite d'événements de violence extrême semble effectivement attester de cette tendance à traverser plusieurs étapes: d'abord "une phase de refoulement qui sera tôt ou tard suivie par une inévitable "anamnèse" ("le retour du refoulé”) et qui peut parfois virer à l'obsession mémorielle » (Traverso 2005 : 44). Dans le cas de l'Insurrection du sud, la configuration se complique. Les conditions "posttraumatiques » n'ont pas offert le moyen de verbaliser le souvenir, de réhabiliter d'autres vérités que celles de l'appareil colonial. Jusqu'aux années 1970, elles ont imposé le silence comme protection contre la peur et l'humiliation, et comme forme de respect du pacte posé au cours du procès. Les témoignages d'aujourd'hui rapportent cette grande période de silence des aînés :

« Il [mon père] nous racontait jamais. Je n'ai jamais entendu parler de ces histoires

là de la part de la bouche de mon papa ».(Constant T., op. cit.)

"Alors moi petite fille, je questionnais notre grand-père. Je disais "Mais papa : que

faisait ton papa, que faisait ta maman ?". Il ne répondait pas. Je me disais "bon". Je 
prenais ça pour une sorte de fermeture d'esprit [...]. Maman n'a jamais raconté. La preuve, c'était toujours un non-dit. Elle nous faisait comprendre que c'était quelque chose de bizarre ».(Yolette J., op. cit.)

«Par exemple, il [mon père] ne m'a jamais dit si Saint-Just [mon grand-père] est né à Haïti, ou si Saint-Just est né en quelle commune en Martinique [...]. Vous voyez, ce côté-là, clos [...]. Le peu de choses qu'il nous disait, ce n'était pas par honte, mais l'affaire est comme ça. On l'étouffe [...]. C'était pas toute la populace qui acceptait n'importe quoi. Mais quand le vécu est vécu, vous ne pouvez changer quoi que ce soit. Mais je me suis toujours posé la question: pourquoi Daniel [frère de SaintJust] ? Pourquoi nous ne sommes pas informés de ce qui s'est passé effectivement [...]. On dit il est mort. Mais comment il est mort, on ne sait pas ».(Louis-Félix B., 80 ans, retraité de la fonction publique, petit-fils du frère de Daniel Bolivard, condamné à mort par contumace, enfui dans l'île de Sainte-Lucie)

La pratique de ce silence instaure la consigne de ne pas dévoiler ce qui peut raviver l'idée d'une faute et qui peut exposer la famille à une vulnérabilité plus grande encore. Il y a dans ce silence, l'impératif de cacher. On est dans la configuration du taire décrite par David Le Breton (1997 : 126-127), avec un silence qui « épargne les membres de la famille » et «vise à prévenir l'intrusion de significations qui risqueraient de percuter les fondements mêmes de leur rapport au monde ».

Mais ce silence, s'il se fait protecteur et défenseur vis-à-vis de l'hostilité extérieure, s'il est une des formes d'adaptation au cadre moral légitimé avec force au cours du procès, il n'est pas pour autant - par cette absence de « rappels » sur l'expérience antérieure l'unique modalité de transmettre le passé. Dans chaque famille, des fragments de paroles ont été délivrés comme s'il s'agissait de ne pas perdre de vue une histoire souterraine qui contredit la version officielle.

\section{Les passeurs de mémoire}

Louis-Félix, comme d'autres, localisent des "silencieux " et regrettent avant tout, au travers de cette parole non délivrée, de n'avoir pas pu rendre plus consistant ce qu'ils savaient par ailleurs. Car Daniel Bolivard, cet oncle lointain né en 1840, trouve bien sa place au sein d'une généalogie transmise que son descendant Louis-Félix décline sans difficulté, connaissant jusqu'aux traits de caractère de l'insurgé disparu : «Il était assez brigand. Il était assez turbulent. » Chacun connaît en effet des morceaux de l'histoire divulgués par les uns ou par les autres. Au silence du père de Constant T. s'oppose la parole de la mère; à celui du grand-père de Yolette J. se substituent les histoires racontées par l'une de ses filles, tante de Yolette. La circulation parvient ainsi à se faire au moyen de "passeurs", ceux qui connaissent plus que les autres, sans que ce statut de dépositaire du savoir familial ne relève d'aucune assignation traditionnelle, ni d'une quelconque initiation formalisée. Elle se produit par l'usage auprès de ceux prédisposés par leur écoute et leur lien avec un autre " passeur » à être ces détenteurs de mémoire, dont certains sont les témoins rencontrés aujourd'hui. C'est le cas de Pierre C., 74 ans, employé retraité de la poste, ayant vécu en métropole et arrière-petit-fils de la branche illégitime des enfants d'Eugène Lacaille. Sa connaissance des parentés du quartier de la Régale - là où se trouvait le camp insurrectionnel - comme des propriétés et de leur transmission, est précise: elle se décline au cours de longues conversations qui confirment la centralité du couple «famille-terre» dans l'univers rural. Pierre se sait « spécialiste » et reconnu comme tel : 
" Il y a beaucoup de choses sur les terrains que je connais, parce que mon grandpère me disait [...]. Ils ne savent pas [...]. C'est moi quand je suis revenu de métropole qui leur ai expliqué [...]. C'était le père, Édouard, qui était le père de son père. Il ne savait pas. Il est plus grand que moi. Il est mort en 80 , avant 80 . Et il ne savait pas [...]. Moi, je le raconte parce que moi, j'ai retenu pas mal de choses. Ils sont plus grands que moi mais ça ne rentrait pas dans leur tête. Moi, j'ai gardé ça, je ne sais pas pourquoi ».(Pierre C., 74 ans, arrière-petit-fils d'Eugène Lacaille)

Les réseaux de la transmission opèrent à des moments particuliers. Pour Pierre, c'est pendant son enfance lorsqu'il est en charge de s'occuper de son grand-père malade :

"J'avais 9 ans, 10 ans [...]. C'est resté dans un coin. C'est parce que c'est intéressant. Quand il [mon grand-père] me racontait ça, eh bien, c'est comme si je raconte ça à ma petite-fille et puis elle retient. [...] J'allais dormir avec lui [...] je faisais sa toilette. Je couchais dans un petit lit à côté et il avait un autre petit lit [...]. Ah oui, il me parlait beaucoup ».(Pierre C., op. cit.)

Pour Liliane F., c'est son père âgé qui transmet l'histoire familiale en des moments quasi ritualisés :

« Il aimait bien parler. Il était très ouvert [...]. Et il avait une mémoire d'éléphant vu son âge puisqu'il avait pratiquement 80 ans quand il m'a raconté ça [...]. J'avais une vingtaine d'années [...]. Il se couchait dans son pliant et puis il racontait. Il racontait les souvenirs d'antan et entre autres choses, les souvenirs de l'insurrection du Sud. Ça revenait souvent, souvent, souvent. Parce que c'est une histoire qui a beaucoup marqué sa vie, dans la mesure où beaucoup de sa famille s'est expatriée y compris son père ».(Liliane F., op. cit.)

21 Grâce à "ces passeurs de mémoire ", de véritables récits du moment insurrectionnel prennent forme. On en retiendra seulement deux ici, rapportés par des témoins dont il est quasi certain qu'ils n'ont eu recours à aucune source écrite pour alimenter les propos qui leur ont été transmis :

«Voilà. Eugène Lacaille, je ne l'ai pas connu. J'ai connu mon grand-père qui m'expliquait tout, qui me disait comment ça s'était passé au temps de Codé tout ça. Parce que le grand-père Eugène Lacaille travaillait à la Mauny [nom de la propriété de Codé] [...]. C'était Codé qui commandait, qui était le propriétaire. [...] Mon grandpère me parlait de Codé, comment ça s'est passé du temps de Codé parce que le père Eugène Lacaille était contre l'esclavage, parce que Codé, il attachait les esclaves pour que les esclaves ils ne partent pas la nuit puisque le lendemain, il y avait besoin de cannes pour faire marcher l'usine. Eugène Lacaille, ça ne lui plaisait pas. Donc il avait monté un petit groupe, un machin comme ça, puisque c'était du temps de l'abolition de l'esclavage [...]. Et puis ils sont montés sur la croix Codé. C'est pour cela qu'on appelle là, la croix Codé, là où l'on a tué Codé [...]. Il m'a raconté que Codé attachait les esclaves avec les pieds pour ne pas partir. Et comme ils ont monté un complot avec les esclaves en groupe [...] on dit qu'il avait son clan. Et il est monté avec son clan, ils ont baré [intercepté] Codé et puis ils l'ont assassiné ».(Pierre C., op. cit.)

Le propos de cet article destiné à identifier des «mémoires qui circulent » ne peut bien évidemment pas inclure l'analyse significative des écarts à la représentation historienne et le contenu symbolique qu'ils marquent, mais on soulignera cependant pour le témoignage qui vient d'être rapporté (et pour d'autres), la localisation de l'événement de 1870 dans la période esclavagiste, ce qui pourrait indiquer non pas l'anachronisme produit par une "mémoire fausse " mais l'appréhension des conditions de l'époque vécues comme un prolongement de l'institution de l'esclavage. Le récit qui suit, forcément très écourté, transmis par une descendante de Jean Vincent-Rosine, présente 
pour sa part une adéquation forte avec des descriptions fournies dans les actes du procès non connus des témoins à l'époque de la collecte de ce récit ${ }^{16}$ :

«On disait que c'était mon arrière-grand-père, mon aïeul [...] qui était chez lui. On est venu le chercher pour lui dire qu'on a attrapé Codé [...]. Je crois qu'il [Codé] avait frappé quelqu'un et nous, on venait se venger. Lorsqu'il a su qu'on venait le chercher pour lui rendre ce qu'il avait fait, il est parti dans les bois. [...] Il y avait tout le monde, toute la population qui était derrière lui. Il est rentré dans les bois [...]. Mais il y a quelqu'un qui avait pitié de lui, qui lui portait à manger comme des cochons [...]. Mais lorsqu'on l'a pris, mon aïeul, il est chez lui. On est venu pour lui dire: "Misié Vincent, nou pren Kodé jodia. Ki sa nou ka fè ?" "'Monsieur Vincent, on a attrapé Codé aujourd'hui. Qu'est-ce qu'on fait ?") [...]. Il est sorti comme ça. "Bon, zot pren Kodé jodia ? Mwen ka monté" ("Vous avez attrapé Codet aujourd'hui ? Je monte"). Il est monté. Lorsqu'il est arrivé, il a dit que si c'était de son temps, il aurait fait quelque chose. Dès qu'il a dit ça, on l'a frappé mais lui-même, il ne l'a pas touché [... ]. C'était un Monsieur, un Monsieur droit dans ses affaires [...]. Tout le monde le considérait comme un grand homme [...]. Et puis on dit que c'est la famille Vincent qui a tué [...]. On l'a accusé [...]. On est venu le chercher pour l'emmener au bourg. [...] On l'a embarqué [...]. En arrivant en prison, il s'est étouffé. Il est mort étouffé [... ]. C'est un arrêt cardiaque qu'il a fait [...]. On ne l'a pas battu, on ne l'a pas touché dans la prison [...]. Il est mort de douleur, de chagrin, de douleur, de révolte». (Denise V., 93 ans, ancienne cultivatrice, arrière-petite-fille de Jean Vincent-Rosine, décédé en prison en 1871)

\section{Le secret protecteur : la mémoire réparatrice}

Le rôle de ces porteurs de parole permet de nourrir une autre vision, forgée en contrebande, qui reconfigure les figures familiales, les «ré-héroïse », sur le mode d'une narration retenue dans l'entre-soi familial. Elle déploie une autre stratégie du silence opposée cette fois-ci à l'extérieur, s'appliquant, par le partage du sens caché, à sceller le lien familial : un «savoir-taire » qui « consolide une position relationnelle en tenant les autres éloignés» (Le Breton 1997: 126). Quelques membres de la famille Vincent dévoilent ce qu'ils considèrent avoir été l'un de ces secrets partagés, celui du sort réservé au patronyme de leur aïeul, Jean Vincent-Rosine. Car l'abandon de la deuxième partie du nom familial (Rosine) n'aurait pas été le fait des descendants directs, les fils de l'insurgé, mais celui de cousins, parents éloignés qui auraient préféré scinder le groupe en deux parties pour se décharger sur l'une du fardeau de l'anathème. "Ma mère aurait dû être mademoiselle Rosine. J'ai l'impression qu'on est dépossédé de quelque chose $\aleph^{17}$. Il est même dit qu'un officier de l'état-civil se serait rendu complice de la tromperie. Le nom intervient ici avec toute sa force symbolique pour attester de l'investissement dont il est le lieu : celui d'ouvrir à la possibilité de l'identité pleinement reconnue dans un réseau de relations qui donnent sens à l'ordre du monde. L'attachement tenu secret à ce nom dont ceux qui le portent refusent qu'il ait été "sali», rejoint l'analyse de Denis-Constant Martin qui, s'appuyant sur l'exemple des États-Unis, rappelle que pour les esclaves, le passage à un système prénom-nom patronymique permettait par «transmission du nom du père aux enfants de signaler dans un honneur recouvré l'existence de familles et de les séparer symboliquement de leurs propriétaires » comme il sera, pour les émancipés, le moyen de dire « la fin de l'asservissement et la liberté retrouvée» (Martin 1998 : 77-78). La polarisation sur le nom patronymique de la part des descendants des insurgés apporte cependant de la nuance quant à la perspective sur les Antilles françaises selon laquelle les noms attribués à l'abolition auraient été d'un usage purement formel (Cottias 2003a : 173). Si les pratiques révèlent effectivement des variations, surtout autour des prénoms et 
surnoms, et sont le "signe d'une histoire non enregistrée » (ibid.), elles se condensent cependant autour de la stabilité désirée d'un patronyme attribué à une lignée de descendants, nous éloignant de l'idée, prégnante dans l'œuvre d'Édouard Glissant, d'une profusion nominative qui masquerait le manque du «Nom ancien» (Degras 1995: 73). Rien de tel dès lors que la famille trouve un ancrage, celui de la terre, et appelle le couplage durable du nom et du lieu pour inscrire l'identité. Il s'agit tout à la fois de conserver le nom, conserver la terre et par-dessus tout, conserver sa place dans la lignée d'un «nom-patrimonial ». Retracer l'histoire de l'atteinte portée au nom, savoir décrire les mutations qu'il a subies, comme chez Yolette J. ou Liliane F., c'est une manière de ne pas perdre l'agencement social qu'il avait pour fonction de stabiliser.

D'autres histoires élaborées contre le dispositif narratif dominant procèdent au même travail de restauration des figures familiales. Elles s'articulent autour de la justification des agissements de leurs aïeux résolument replacés dans la trame du conflit structurant de la société martiniquaise, celui où s'énonce clairement l'oppression raciale du fond esclavagiste. Nous avions déjà entrevu cette trame explicative avec le témoignage de Pierre C. pour qui Eugène Lacaille avait levé son « clan » contre l'esclavage. Elle parcourt d'autres récits transmis par les quelques dispensateurs de paroles, récupérés et aménagés dans une geste réparatrice face à une violence dont seul l'imaginaire semble avoir été en mesure de dénoncer la portée :

«Il faut dire que Codé n'était pas un saint. Quand je dis un saint, il a fait pas mal d'atrocités [...]. Alors comme tout le monde le craignait, ce n'était pas la peine de chercher à comprendre [...]. Il n'y avait pas une revanche mais une vengeance. Il fallait se venger quand même [...] parce qu'il faisait partie des gens qui jugeaient les nègres, des gens qui jugeaient les esclaves [...]. Il était d'une extrême sévérité paraît-il. Il n'était pas aimé du tout [...]. Je n'ai jamais eu honte. Je me suis dit, “bon, eh bien, c'est une histoire" [...]. Il y a tellement d'histoires. Même pas sur Codé seulement, tellement d'histoires sur l'esclavage, tellement de choses qui se passaient. Pour moi, c'était pas naturel [d'avoir tué Codé] mais enfin, c'était pas très émouvant ».Jules V., 68 ans, ancien employé RATP, puis agriculteur, arrière-petitfils de Jean Vincent-Rosine)

"Codé avait des servantes à la maison, et il avait des esclaves aussi. Il les obligeait à manger sous le fouet des repas qu'on avait préparés, mélangés à des excréments humains. Ça, je ne sais pas si c'est répété, mais mon père me l'a dit. Mon père m'a dit que son père lui a dit ça. Donc, il avait exacerbé tout le quartier, tous les noirs [... ]. C'était un vieux béké méchant [...]. Donc, ses oncles et mon arrière-grand-père ont participé aussi à la descente qu'il y a eu chez Codé. On a brûlé sa maison. On l'avait trouvé caché dans un parc à cochon, relativement loin de la maison. On l'a ramené. On l'a exécuté [...]. Par contre, je suppose qu'au témoignage de mon père, que son grand-père faisait partie des gens qui donnaient les ordres [...]. Et que ça a été fait et proprement fait par les gens de l'insurrection. On ne donne pas les noms. Mais, c'est un truc qui a été fait. J'ai entendu ça de la bouche de mon père, il y a très longtemps [...]. À l'époque, les rapports que l'on avait avec les autorités, c'étaient des rapports de crainte [...]. Et d'avoir osé braver une autorité pour diriger une insurrection, diriger des hommes pour braver la milice, la gendarmerie, les armes, ça fait tout un passé lourd, trop lourd pour ceux qui viennent derrière [...]. Mon père n'a jamais eu honte de quoi que ce soit. Et mon père nous a toujours incités à admirer notre grand-père [...]. Il nous racontait cette histoire avec, je ne vais pas dire de la joie, mais de la fierté [...]. Il avait besoin d'être entendu mon père [...]. Mon père s'est dégagé de sa souffrance en nous racontant [...]. Il ne le cachait pas. Et je pense qu'il le racontait souvent parce qu'il y a eu beaucoup de souffrance pour lui et pour sa famille. Mais aussi parce qu'il voulait éveiller en nous ce sens de l'honneur [...]. J'en suis fière parce que lui, il n'était pas instruit. Mais il a su mettre 
cette estime en nous pour mon arrière-grand-père, et cette admiration aussi. Et je

sens que j'ai ce petit sang révolutionnaire en moi aussi ».(Liliane F., op. cit.)

\section{Temporalités des mémoires}

25 La récupération de ces récits dans l'actualité mémorielle ne peut être ignorante des temporalités qui interviennent dans leur élaboration, une ignorance qui les ferait être comme la preuve d'une mémoire suspendue dans le temps, reconduite pareille à ellemême. On a déjà suggéré les étapes de la venue au dicible d'un événement traumatique par les générations successives. Sans en développer plus la teneur, il faut cependant souligner ici avec force le travail accompli par chacun des "porteurs/créateurs" de mémoire en fonction des ressources rendues disponibles selon les périodes. Les constructions mémorielles dont il est question, nous l'avons vu, sont enchâssées dans le jeu des rapports de pouvoir que rappelait Michel-Rolph Trouillot (1995). Elles ont longtemps été soumises à ce régime de visibilité où des "mémoires fortes", celles officielles entretenues par des institutions, affaiblissent les manifestations de leur existence, les rendant «souterraines, cachées ou interdites» (Traverso $2005: 54)$. Elles sont des "couches stratifiées selon le temps" qui se composent et se superposent en fonction de la marge que leur accorde le «temps politique» (Boursier 2004: 34). Les souvenirs de l'insurrection se modifient au fil de ces temporalités successives liées autant à la capacité des « témoins » à en énoncer la teneur d'une expérience douloureuse qu'à la possibilité offerte par le cadre socio-politique pour en modifier le contenu et le statut. La frénésie mémorielle entamée à Rivière-Pilote bien avant celle de l'espace national français, avec une première commémoration officielle de l'insurrection en 1984 et bien d'autres manifestations d'une « contre-mémoire » développées par la suite, a autorisé des aménagements nouveaux et permis le passage au dicible. Sans compromettre l'identification des «souvenirs remémorés » de Paul Ricœur (2000), ce travail conforté par le langage nouveau des autorités locales, puis nationales, a modifié les contenus narratifs. Certains des témoins, pas tous, se livrent aujourd'hui à une véritable quête pour attester, vérifier, compléter les versions recueillies de leurs histoires. Ils datent et racontent d'eux-mêmes l'apparition de ce nouveau régime. Ils identifient la transformation qu'il a introduite dans les mémoires qui leur avaient été léguées, en mettant à disposition de nouvelles ressources, hors du champ familial, et la conçoivent par-dessus tout comme une possibilité de réhabilitation de la trajectoire familiale. $\mathrm{Ni}$ entrepreneurs de mémoire, ni militants, certains de ces descendants d'insurgés rencontrés s'inscrivent plutôt, à partir de cette transformation, dans une sorte de continuité renouvelée vis-à-vis d'un désir familial tendu vers la restauration d'un corps blessé. Ce travail de quête élargie montre l'interférence de la mémoire collective et de la mémoire historique, sans que la présence de la deuxième soit la marque de l'existence révolue de la première, mais l'incorporation de l'une à l'autre, dans un contexte où la circulation mémorielle continue de sceller le lien social. 


\section{Retour conclusif sur le phénomène mémoriel aux Antilles françaises}

$26 \mathrm{Au}$ terme de cette exploration, on n'hésitera pas à affirmer, pour la Martinique, l'existence de mémoires collectives correspondant aux perspectives offertes à la fois par Paul Ricœur et Maurice Halbwachs, à savoir la formation de récits s'appuyant sur des images relatives à des faits antérieurs attestant, dans le dédale de la reformulation fictionnelle, que "quelque chose s'est produit». Ces récits circulent de manière "vivante » à travers l'architecture intergénérationnelle. Ce constat d'apparence assez banal fait apparaître ce qui était tenu jusque-là pour absent, à savoir des "témoins ", certes indirects, mais des témoins tout de même, au sens où ils apportent, non pas tant la voix humaine d'une expérience vécue qui s'opposerait à l'archive historienne, comme on l'entend habituellement (Wieviorka 1998: 168), mais des discours où le référent explicite devient l'événement passé. Parler de témoins au sens premier du terme, n'a de signification pour les Antilles françaises qu'avec la contrepartie qu'il suggère : la sortie de tout ce qui est à la fois de l'implicite, de la formulation inachevée ou en devenir, de la dispersion dans les méandres de mémoires détournées. Car l'explicite du référent du conflit entre maîtres et esclaves au travers de l'expression d'un souvenir vivant, est bien tenu pour l'autre grand absent des configurations mémorielles des Antilles françaises. Ce n'est pas un hasard si l'on doit à Richard Price (2000) d'avoir interpellé sur le niveau de conscience toujours possible à débusquer à la Martinique, en résistance aux dispositifs censés l'anéantir. L'anthropologue n'a pas hésité à nommer " historiens ", les dépositaires de la mémoire des "Premiers Temps» de l'épopée du marronnage dans l'ancienne Guyane hollandaise, allant jusqu'à restituer les résultats de sa recherche sur le mode continu d'une double entrée - celle des récits collectés; celle de son travail anthropologique et archivistique - pour mieux montrer que la mémoire collective en question est "histoire». Elle construit des récits en rapport avec l'antériorité d'événements qu'elle relate sur le mode d'une érudition critique auquel l'historien n'a en définitive rien à envier (Price 1994).

Si les récits qui circulent sur l'Insurrection du sud n'atteignent pas un tel niveau de sédimentation, ils offrent cependant des parallèles troublants avec ceux décrits par Richard Price, notamment dans les formes empruntées par le savoir, celles des « histoires toute simples »; des «fragments généalogiques »; « des listes de terre »; « des suites de noms » (ibid. : 19). Dans tous les cas, ils formulent eux aussi une connaissance du passé qui n'élude pas ce que l'on appellera par raccourci « le réel », sachant que celui-ci est toujours le moment d'expression d'imaginaires singuliers, capables pour certains de s'institutionnaliser, ici l'imaginaire colonial imposant la puissance de sa vision de la suprématie raciale. Le « réel » qui nous est livré au cours des récits des insurgés épouse différents niveaux de pertinence. Ils renvoient au souvenir de l'antériorité d'un passé issu de cette expérience cognitive dont parle Ricœur (2000: 59) et qui rend la chose souvenue en tant que « donnée à nouveau », parce que résultant de sa perception préalable. S'il ne fait pas de doute que ces souvenirs relatés ont bien pour référent explicite le « réel » de l'insurrection et sa violence ultime, ils traduisent aussi d'autres "réels", ceux où s'entremêlent les représentations successives des générations ayant en commun cette "scène primitive " à la manière dont Eyerman (2008: 15) parle de l'esclavage pour l'identité africaine-américaine, scène réitérée dramatiquement lors de l'échec de la 
Reconstruction et dont il affirme qu'elle est au fondement d'une "culture collective du traumatisme ». Sans l'avoir vécue, les générations d'aujourd'hui ayant en partage le souvenir de l'insurrection, continuent d'être identifiées par cette scène primordiale et de s'identifier à travers elle, tout en montrant au fil d'aménagements continus dus à l'éloignement temporel, le jeu possible des écarts à l'ordre symbolique dominant.

Ces mémoires des descendants des insurgés tenues jusque-là dans un anonymat voué à l'inexistant alors qu'elles étaient rivées aux noms qui les habitent, révèlent bien la conscience d'un moment colonial particulièrement oppressif et de ses conséquences. Elles disent la véracité de représentations évolutives issues de la réception tout à la fois intériorisée et contestée de la vision imposée par l'appareil de la domination légitime. Mais plus que tout, dans le cadre de ce propos, elles renseignent sur la présence de narrations ayant «le réel » pour point d'accroche et le sensible de l'expérience comme mode d'assemblage entre hier et aujourd'hui.

\section{BIBLIOGRAPHIE}

BASTIDE, R., 1967, Les Amériques Noires, Paris, Petite Bibliothèque Payot.

BERTRAND, R., 2006, Mémoires d'empire. La controverse autour du « fait colonial », Paris, Éditions du Croquant.

BOURSIER, J.-Y., 2004, « D'une mémoire à l'autre », in V. BONNET (dir.), Conflits de mémoire, Paris, Karthala : 33-44.

CHIVAllon, C., 1998, Espace et identité à la Martinique. Paysannerie des mornes et reconquête collective (1840-1960), Paris, Éditions du CNRS.

- 2005a, « Résurgence des mémoires de l'esclavage : entre accélération généralisée et historicité singulière », Diasporas, Histoire et Sociétés, 6 : 144-155.

- 2005b, « L'usage politique de la mémoire de l'esclavage dans les anciens ports négriers de Bordeaux et Bristol », in S. DUFOIX \& P. WEIL (dir.), L'esclavage, la colonisation et après..., Paris, PUF : 533-558.

- 2009, « La diaspora noire ou l'ailleurs impossible. À propos de l'Insurrection paysanne de 1870 à la Martinique », Diasporas, Histoire et Sociétés, $13: 80-109$.

- À paraître, « Mémoires de l'esclavage et actualisation des rapports sociaux », in A. MENDES ET AL. (dir.), Les traites, les esclavages et leurs productions sociales et culturelles (XIV $\left.{ }^{e}-X X I^{e}\right)$. Recherches francophones, Paris, Éditions CNRS.

COMITÉ POUR LA MÉMOIRE DE L'ESCLAVAGE, 2005, Mémoires de la traite négrière de l'esclavage et de leurs abolitions, Paris, La Découverte.

CONSEIL DE GUERRE, 1871 Martinique, Insurrection du Sud (22 septembre 1870), Première et deuxième séries, Paris, BNF, (LK12-1232).

COQUIO, C., 2008, « Retours du colonial ? », in C. COQUIO (dir.), Retours du colonial. Disculpation et réhabilitation de l'histoire coloniale, Nantes, L'Atalante : 9-43. 
COTTIAS, M., 2003a « Le partage du nom. Logiques administratives et usages chez les nouveaux affranchis après 1848 », Cahiers du Brésil contemporain, 53-54 : 163-174.

- 2003b, « Le silence de la nation. Les "vieilles colonies" comme lieu de définition des dogmes républicains ", Outre-Mers, 90 (338-339) : 21-45.

- 2006, « Oubli, pardon et ressentiment : la citoyenneté à la Martinique (1848-1850)», in M. COTTIAS, A. STELLA \& B. VINCENT (dir.), Esclavage et dépendances serviles : une histoire comparée, Paris, L'Harmattan : 147-162.

- 2007, « Ces "hommes dangereux" de 1848. L'amnistie à l'épreuve de l'abolition de l'esclavage de 1848 », Genèses, $66: 30-50$.

DEGRAS, P., 1995, « Nom des pères, histoire du Nom : Odono pour mémoire », Études créoles, 18 (2) : 69-81.

EYERMAN, R., 2008, [2001] Cultural Trauma. Slavery and the Formation of African American Identity, Cambridge, Cambridge University Press.

FASSIN, D. \& RECHTMAN, R., 2007, L'empire du traumatisme. Enquête sur la condition de victime, Paris, Flammarion.

GIRAUD, M., 2005, « Les enjeux présents de la mémoire de l'esclavage », in P. WEIL \& S. DUfoIX (dir.), L'esclavage, la colonisation et après, Paris, PUF : 533-558.

GLISSANT, É., 1981, Le discours antillais, Paris, Éditions du Seuil.

- 2007, Mémoires des esclavages, Paris, Gallimard.

HALBWACHS, M., 1997, [1950] La mémoire collective, Paris, Albin Michel.

JOLIVET, M.-J., 1987, « La construction d'une mémoire historique à la Martinique : du

Schoelchérisme au Marronisme », Cahiers d'Études africaines, XXVII (3-4), 107-108 : 287-309.

KANSTEINER, W., 2002, « Finding Meaning in Memory : A Methodological Critique of Collective Memory Studies », History and Theory, $41: 179-197$.

LE BRETON, D., 1997, Du silence, Paris, Métaillé.

- 2006,Anthropologie de la douleur, Paris, Métailié.

MARTIN, D.-C., 1998, « Le poids du nom. Culture populaire et constructions identitaires chez les "Métis" du Cap », Critique internationale, 1 : 74-100.

MENCHE DE LoISNE, C., 1931, [1871] « Insurrection de la Martinique, 22 Septembre- $1^{\text {er }}$ Octobre $1870 »$, Revue de la Martinique, $17: 1-39$.

MULOT, S., 2000, «Je suis la mère, je suis le père! » L'énigme matrifocale, Thèse de doctorat, Paris, EHESS.

- 2007, « Le mythe du viol fondateur aux Antilles françaises », Ethnologie française, XXXVII (3) : 517-524.

PAGO, G., 1974, Histoire antillaise. L'Insurrection du Sud, contribution à l'étude sociale de la Martinique, Université Antilles-Guyane, Groupe universitaire de recherches inter-caraïbes, Études et documents, 14 .

PRICE, R., 1994, Les premiers temps. La conception de l'histoire des Marrons Saramaka, Paris, Éditions du Seuil.

- 2000, Le bagnard et le colonel, Paris, PUF. 
REINHARDT, C. A., 2006, Claims to Memory. Beyond Slavery and Emancipation in the French Caribbean, New York, Berghahn Books.

RICcUR, P., 2000, La mémoire, l'histoire, l'oubli, Paris, Éditions du Seuil.

SCHMIDT, N., 1999, «Commémoration, histoire et historiographie. À propos du 150e anniversaire de l'abolition de l'esclavage dans les colonies françaises », Ethnologie française, XXIX, 3 : 453-460.

SCHOELCHER, V., 1872, L'arrêté Gueydon à la Martinique et l'arrêté Husson à la Guadeloupe, Paris, Le Chevalier.

scoTT, D., 1997, « “An Obscure Miracle of Connection” : Discursive Tradition and Black Diaspora Criticism », Small Axe, $1: 19-38$.

STORA, B., 2007, La guerre des mémoires. La France face à son passé colonial, Entretiens avec Thierry

Leclère, La Tour d'Aigues, Éditions de l'Aube.

TEVANIAN, P., 2007, La République du mépris, Paris, La Découverte.

TRAVERSO, E., 2005, Le passé, modes d'emploi, Paris, La fabrique éditions.

TROUILlOT, M.-R., 1995, Silencing the Past. Power and the Production of History, Boston, Beacon Press.

VERGÈS, F., 2006, La mémoire enchaînée. Questions sur l'esclavage, Paris, Albin Michel.

WIEVIORKA, A., 1998, L’ère du témoin, Paris, Hachette.

YANG-TING, J., 2000, Le mouvement indépendantiste martiniquais, Petit-Bourg, Ibis Rouge Éditions.

\section{NOTES}

1. Voir entre autres l'analyse de Romain BERTRAND (2006: 206) qui envisage le débat mémoriel actuel avec ses «dynamiques concurrentielles de production militante des identités victimaires » comme relevant « d'une logique de fonctionnement ordinaire du système politique ».

2. Sur cette "guerre des mémoires» relative au fait colonial, voir sTORA (2007). Sur le "soupçon » et sa place dans la nouvelle économie morale contemporaine, se reporter à Didier FASSIN et Richard RECHTMAN (2007: 414) pour qui l'usage de la catégorie « traumatisme » aujourd'hui largement admise traduit un jugement moral qui sélectionne "les "bonnes" et les "mauvaises" victimes " selon des «échelles de légitimité parmi les victimes ». Voir aussi l'analyse de Pierre tevanian (2007: 50-77) selon laquelle le débat mémoriel actuel relatif à l'esclavage et à la colonisation a reconduit des formes de discrimination en disqualifiant certains discours.

3. L'article récent de Catherine coQuio (2008: 11) rend compte de cette «conjoncture singulière » qui soumet le mouvement de reconnaissance des crimes coloniaux à une « contre-offensive » et à une « résurrection de l'utopie coloniale » au plus haut niveau de l'État.

4. On pense ici en particulier aux activités du «Comité Marche du 23 mai » créé à la suite de la marche silencieuse du cent-cinquantenaire de l'esclavage qui a réuni 40000 personnes à Paris en 1998 et dont l'objectif est d'aider les originaires des Antilles, de la Guyane et de la Réunion à assumer leur identité de Français descendants d'esclaves. Voir le site : <http://www.cm98.fr/>. 
5. Par l'expression de « vindicte anti-mémorielle », il s'agit de faire référence au contexte actuel suggéré plus haut (notes 2 et 3 ) où la mémoire relative à l'esclavage se voit comme pliée à l'exigence d'être plus légitime ou plus « vraie » encore que d'autres revendications mémorielles.

6. Pour une analyse des significations de l'usage actuel du registre mémoriel relatif à l'esclavage, voir CHIVALLON (2005a).

7. Sur l'usage de la symbolique de l'abolition lors du cent-cinquantenaire, se reporter à l'analyse comparative des dispositifs mémoriels mis en place dans les anciens ports négriers en France et en Angleterre (CHIVALLON 2005b). Sur la place de l'esclavage dans le récit républicain, voir COTTIAS (2003b) et VERGÈs (2006). Il revient à l'historienne Myriam Cottias d'avoir ouvert le véritable chantier sur la politique de l'oubli aux Antilles françaises.

8. Dans le cadre de cet article, on se limitera à résumer à grands traits le déroulement de cette insurrection dont une analyse plus complète est fournie dans CHIVALLON (2009).

9. Les Békés forment le groupe des Blancs descendants des maîtres esclavagistes. Le terme « caste » appartenait au vocabulaire de l'époque.

10. Le procès des insurgés a été placé sous la responsabilité d'un Conseil de Guerre et d'un tribunal militaire. Au cours de cet article, toutes les mentions faites à ce procès concernent la référence CONSEIL DE GUERRE (1871). On trouvera la localisation précise de ces mentions - non reprises ici dans le détail pour ne pas alourdir le texte - dans CHIVALLON (2009).

11. Sur la représentation du «viol originel » dans les cultures antillaises, se reporter à GLISSANT (1981) et surtout à MULOT $(2000,2007)$.

12. «L'Habitation » est le nom donné à la plantation aux Antilles françaises.

13. L'amnistie est proclamée dès le 30 septembre 1870 par le gouverneur et propose un « marché » dans les termes suivants : "À l'exception des chefs de bande et des assassins, j'ordonne de cesser toute poursuite contre ceux qui ont pris part à ces attentats et qui n'ont pas encore été arrêtés, mais à une condition, c'est qu'aucun nouvel attentat contre les personnes ou les propriétés ne sera commis» (CONSEIL DE GUERRE, 1871, $1^{\text {re }}$ série, p.122). L'amnistie est finalement appliquée dans le même esprit au cours du procès à ceux censés avoir naïvement suivi des meneurs dangereux. Outre sa profonde portée symbolique, cette mesure vient aussi répondre à un objectif que le gouverneur ne cachera pas : ramener la population au travail (MENCHE DE LOISNE 1931 : 21-22).

14. Je remercie chaleureusement mon ami Hervé Zénoki, bibliothécaire de Rivière-Pilote, compagnon de cette recherche, qui a fait le lien entre les membres des familles porteuses du patronyme que je recherchais (ou liées à lui) et moi-même. Les rencontres ont concerné un effectif bien plus élevé de personnes: on fait référence ici à celles que la tradition anthropologique nommerait des « informateurs», personnes avec qui des liens soutenus ont été créés.

15. Alfred Marie-Jeanne est la figure emblématique du Mouvement indépendantiste martiniquais (MIM), aujourd'hui président du Conseil régional de Martinique. Sur l'histoire du MIM, voir YANG-TING (2000).

16. J'ai déposé moi-même à la bibliothèque de Rivière-Pilote un double de ces documents provenant de la Bibliothèque nationale de France : aucun de mes interlocuteurs n'y avait eu accès auparavant. 


\section{RÉSUMÉS}

\section{Résumé}

Partant de l'analyse de la situation mémorielle actuelle, cet article diagnostique la question qu'elle fait naître pour la mémoire relative à l'esclavage dans les Antilles françaises, prise dans une frénésie qui renforce l'idée de mémoires soudainement inventées. Des mémoires de l'esclavage de l'ordre de la circulation intergénérationnelle sont-elles présentes ? Cette question renvoie au débat anthropologique propre à ces sociétés où la mémoire collective relative à l'esclavage a longtemps été tenue pour être absente. À partir d'une recherche conduite sur un événement ancien qui reconduit le cadre du conflit originel entre maîtres et esclaves, à savoir l'insurrection de 1870 à la Martinique, l'article décrit les processus mémoriels qu'il a générés. Le recueil de témoignages auprès des descendants des insurgés rend compte de la présence de récits familiaux transmis jusqu'à aujourd'hui selon des modalités particulières liées aux conséquences de la répression de l'événement insurrectionnel. L'interprétation qui s'en dégage amène à revoir la question de la mémoire collective à la Martinique.

\section{Abstract}

Taking the analysis of the current memory situation as a starting point, this article analyses the question that it provokes about the memory of slavery in the French West Indies, subjected to a frenzy that reinforced the notion of spontaneously invented memories. Is there an intergenerational dissemination of slavery memory? This question refers to the anthropological debate in societies where collective memory about slavery has long been deemed absent. Using research carried out on an historical event that revives the framework of the original master/ slave conflict, namely the 1870 insurrection in Martinique, we describe the memory processes this generated. The collection of accounts from descendents of the rebels shows that family accounts have been transmitted until this day, using specific forms related to the consequences of the repression of the rebellion. The subsequent interpretation leads us to review the question of collective memory in Martinique.

\section{INDEX}

Keywords : French West Indies, Conflict, Slavery, Memory Accounts, Transmission

Mots-clés : Antilles françaises, Martinique, conflit, esclavage, mémoires, récits mémoriels, transmission

\section{AUTEUR}

\section{CHRISTINE CHIVALLON}

Centre d'Étude d'Afrique Noire, IEP de Bordeaux. 